\title{
Red Blood Cell Response to Blast Levels of Force Impartations Into Freely Moveable Fluid Surfaces Inside a Closed Container
}

\author{
David Smith ${ }^{1}$, Robert Franco ${ }^{2}$, Christopher A. DiCesare ${ }^{1}$, Daniel K. Schneider ${ }^{1,3}$, \\ Chuck McGill ${ }^{4}$, Quinton D. Smith ${ }^{3,5}$ and Gregory D. Myer ${ }^{1,6,7 *}$
}

${ }^{1}$ Division of Sports Medicine, Cincinnati Children's Hospital Medical Center, Cincinnati, OH, United States, ${ }^{2}$ Department of Internal Medicine, Hematology and Oncology, College of Medicine, University of Cincinnati, Cincinnati, OH, United States, ${ }^{3}$ College of Medicine, University of Cincinnati, Cincinnati, OH, United States, ${ }^{4}$ Laboratory Services, Reid Health, Richmond, IN, United States, ${ }^{5}$ Center for Autoimmune Genomics and Etiology, Cincinnati Children's Hospital Medical Center, Cincinnati, $\mathrm{OH}$, United States, ${ }^{6}$ Departments of Pediatrics and Orthopaedic Surgery, University of Cincinnati, Cincinnati, $\mathrm{OH}$, United States, ${ }^{7}$ The Micheli Center for Sports Injury Prevention, Waltham, MA, United States

OPEN ACCESS

Edited by:

Federico Giove,

Centro Fermi-Museo Storico della

Fisica e Centro Studi e Ricerche

Enrico Fermi, Italy

Reviewed by:

Zsolt J. Balogh,

University of Newcastle, Australia

Ronald Sluyter

University of Wollongong, Australia

Dmitry A. Fedosov,

Forschungszentrum Jülich, Helmholtz-Gemeinschaft Deutscher Forschungszentren (HZ), Germany

*Correspondence:

Gregory D. Myer greg.myer@cchmc.org

Specialty section: This article was submitted to

Biomedical Physics,

a section of the journa

Frontiers in Physics

Received: 09 March 2018

Accepted: 30 July 2018

Published: 21 August 2018

Citation:

Smith D, Franco R, DiCesare $C A$, Schneider DK, McGill C, Smith QD and Myer GD (2018) Red Blood Cell

Response to Blast Levels of Force Impartations Into Freely Moveable

Fluid Surfaces Inside a Closed

Container. Front. Phys. 6:93.

doi: 10.3389/fphy.2018.00093
Background: Blast waves have plagued mankind for centuries, yet their interaction with blood has been largely overlooked. Recent studies of ways to mitigate traumatic brain injury (TBI) in sport have utilized slosh-reducing techniques with varying success. However, hydrodynamic principles have not been used to assess the interaction of intense blast waves and the red blood cells themselves.

Objective: To establish the benefits of slosh reduction by analyzing the degree of damaging effects from a blast wave imparted on human red blood cells both fully and partially contained by rigid surfaces.

Methods: Approximately $40 \mathrm{~mL}$ of blood was collected from 13 males and 25 females ages 18-55 and aliquoted into three separate steel containers (5 cc- control, 15 cc- full and $10 \mathrm{cc}$-partially filled) in preparation for blast testing. An improvised explosive device (IED) blast level model (Vandenberg) was developed by using a nail gun plunger to impart approximately $150 \mathrm{kPa}$ of pressure over a 4 to $6 \mu \mathrm{s}$ timespan into a target container. Blood in steel containers, with and without IED blast exposure, was assayed for cell hemolysis products including serum free hemoglobin $(\mathrm{HgB})$, lactate dehydrogenase $(\mathrm{LDH})$, and potassium (K-ABL) when the containers were fully-filled or partially filled (no-slosh versus slosh). Hemolysis products were measured 1, 4, 24, 48, and $72 \mathrm{~h}$ after blast exposure.

Results: In both the slosh and no-slosh groups, hemolysis increased significantly at each time point after IED blast energy impartation. However, the no-slosh blood samples had less hemolysis. Compared to the slosh group, blood from the no slosh group contained less extracellular HgB ( $p<0.0001)$, lactate dehydrogenase $(\mathrm{LDH})(p<0.0001)$, and potassium $\left(\mathrm{K}^{+}\right)(p<0.0001)$ at all time points.

Conclusions: Damage to human blood resulting from the impartation of a force similar to an IED blast was mitigated by fully containing blood within a volume and thereby reducing fluid slosh.

Keywords: red blood cell, blast exposure, cavitation, blast injury, brain injury 


\section{INTRODUCTION}

Blast wave energy impartation into human tissues has been a topic of concern since the Chinese invented gun powder in 300 $\mathrm{AD}$ and eventually used it in fireworks and weapons [1]. The interaction of these energies with the human crania, resulting in traumatic brain injury (TBI), has in recent decades attracted more attention, especially in the setting of improvised explosive devises (IEDs). Although one can concentrate primarily on kinetic (blast) energy impartation, there are several other forms of imparted energies (thermal, vibrational and acoustic) that have been more extensively studied and could also contribute to tissue damage. Specifically, in an attempt to define the mechanism of energy impartations into human tissues, much has been written about acoustic energy impartations into tissues (such as diagnostic and therapeutic ultrasound). In furthering the understanding of the pathophysiology of blast wave interaction with tissues, the molecular and cytological makeup of the tissue itself may actually be more important than the mechanics of the energy (blast) wave [2]. For example, the International Society of Radiology asserts that when directing ultrasound waves into human tissues, a near total reflection $(99.9 \%)$ of acoustic wave energy from boundaries occurs between gas and tissues, yet once the energy passes into soft tissues (blood in the current project), there is only $0.2 \%$ reflection (a 500 -fold difference). Further, upon comparing specific tissues, the acoustic impedance (the interaction of the tissue to the wave itself) of static blood is nearly identical to brain (1.61 vs. 1.59 Rayl) [2].

The study of imparting energies into human blood is facilitated by the ready availability of blood samples. In addition, blood can be easily tested for apoptosis and lysis (electrolytes and hemoglobin), making this media particularly suitable for the study of blast energy effects on tissues [3]. Blood also is fluid, and thus exhibits slosh. The hydrodynamic study of slosh defines the characteristics of liquids within moving containers [4]. A better understanding of the physics of slosh as it applies to blood within the cranium led to the creation of a gentle jugular compression collar to increase the impedance of the jugular vascular tree. This was hypothesized to divert more flow to the vertebral veins and other capacitance vessels that have higher vascular resistance, which then distend [5] and repackage the cranial space with less potential movement.

Two clinical studies [6, 7] have used a jugular compression collar to reduce cranial slosh. They both demonstrated marked protective effects in high school athletes during competitive fullcontact play with exposure to impacts resulting in accelerations up to $200 \mathrm{~g}$. Initial animal studies also lent support to the physiology of using a small jugular compressive collar in rats by providing an $83 \%$ protective effect when the animals were exposed to a $900 \mathrm{x} \mathrm{g}$ impact model [8].

Here we extend the above work (that used force levels typically experienced on an athletic field) to evaluate blood consequences that may be relevant to battlefield blast wave forces. Specifically, the current study examines the effects of higher order blast wave energy impartations into human tissues (specifically blood) in the setting of slosh reduction techniques. The specific aims of this study include: evaluating both the blood cell response to blast levels of force impartations into freely moveable fluid surfaces inside a closed container (i.e., hydrodynamic force impartationslosh) and the effects of containment of these moving fluids by filling the container. We hypothesized that there would be less blood damage if slosh energy absorption potential was decreased by completely filling the containers prior to the blast. The extent of damage was determined by monitoring for sensitive signs of red blood cell hemolysis including plasma free hemoglobin $(\mathrm{HgB})$, potassium $(\mathrm{K}-\mathrm{ABL})$, and lactate dehydrogenase $(\mathrm{LDH})$ [9].

\section{METHODS \\ Study Participants}

13 males and 25 females ages 18-55 visited the Reid Health Laboratory for blood collection. Each individual reviewed and signed the Institutional Review Board consent form to participate in the study (IRB 2014-1087). Subjects were then asked to complete a short questionnaire to assess their current general physical health status.

\section{Blood Collection}

Approximately $40 \mathrm{~mL}$ of blood was obtained via venipuncture with size 21 needles into heparin with a final concentration of 15 units $/ \mathrm{ml}$. The blood was drawn into and remained in in heparinized vacutainers at room temperature until use. The blood was then delivered into three steel Crossman $12 \mathrm{~g} \mathrm{CO}_{2}$ cartridges: (5 cc- control, $15 \mathrm{cc}$ - full, and $10 \mathrm{cc}$-partially filled) equipped with a threaded acorn nut to completely seal the container. Before transfer to the Blood Blasting Region outside of the hospital, each container was color coded and numbered.

\section{Blood Blasting}

The blasting technique used here was a modification of the IED blast level model [10] (Vandenberg) that used a nail gun plunger in front of rat skulls. This mechanism imparted an approximated maximum $150 \mathrm{kPa}$ over-pressure (within a 4-6 $\mu \mathrm{s}$ timespan) into a target container, a level of energy approximately equal to an IED blast $[10,11]$. To withstand the large forces imparted, steel containers of blood were used for energy impartation and observed for differences in cell damage when the cartridge was filled versus partially filled (no-slosh vs. slosh). The blood blasting procedure was carried out according to the Dewitt Protocol for blast wave impartation (Figures 1-4). The operator was fitted with eye protection, earmuffs, and heavy protective gloves. A black powder Hilti DX550 nail gun was loaded with a red 0.27 caliber powder charge and no projectiles were placed inside the firing barrel. A rubber stopper was secured on the far end of the barrel to assure proper orientation with respect to the $\mathrm{CO}_{2}$ cartridge. The appropriate steel blood cartridge was then brought into place and set "acorn nut down" in the Landmark ${ }^{\mathrm{TM}}$ steel reinforced chamber with the rounded end upwards (Figure 3). A metal bracket was then secured onto the Landmark ${ }^{\mathrm{TM}}$ apparatus and a washer was placed over the opening to disengage the safety mechanism of the nail gun allowing it to fire. The cartridge was then struck with the nail gun plunger, creating a dent and imparting a maximum pressure of $150 \mathrm{kPa}$ within a $5-\mu$ s pressure 


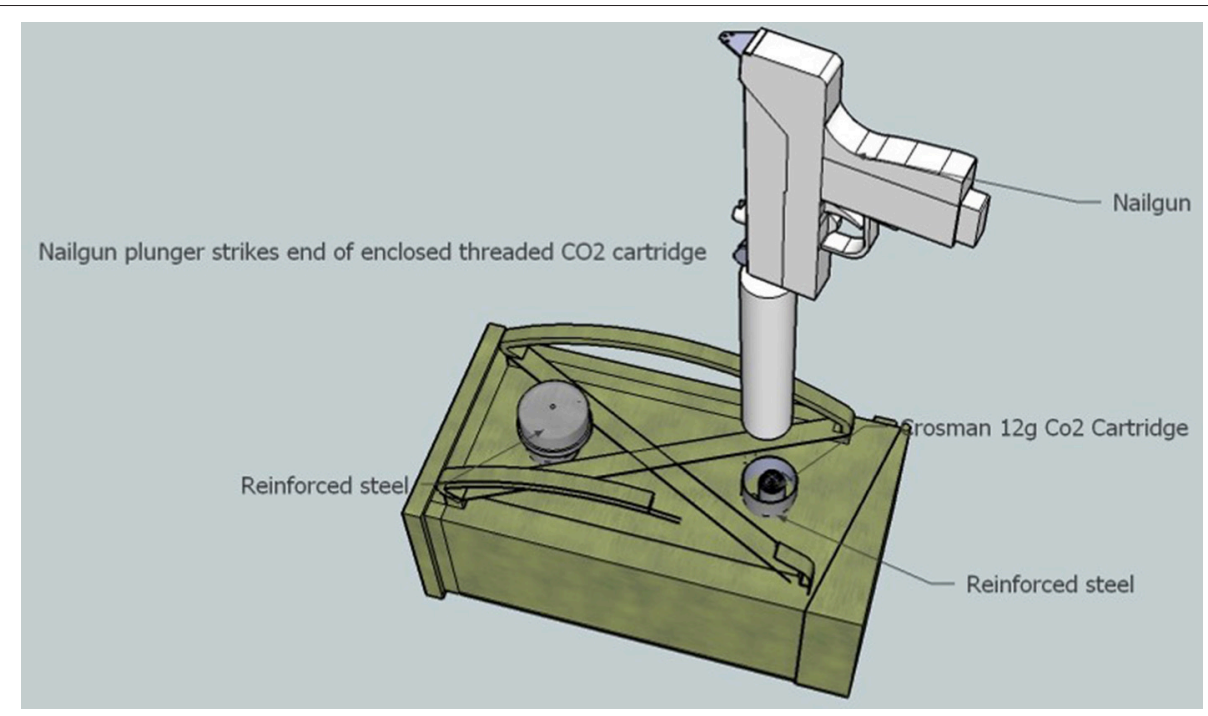

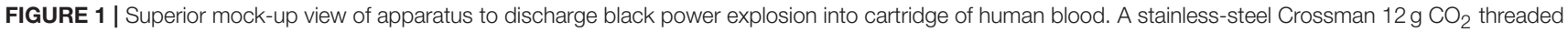
cartridge which contains each sample of human blood was sealed with an nickel plated steel acorn nut was place within a reinforced steel pipe (blast chamber) and capped off to protect the operator from the explosive nail-gun force. The nail gun was positioned above the blast chamber. The chamber was then encompassed in a wooden encasement to position the two chambers in an upright parallel state.

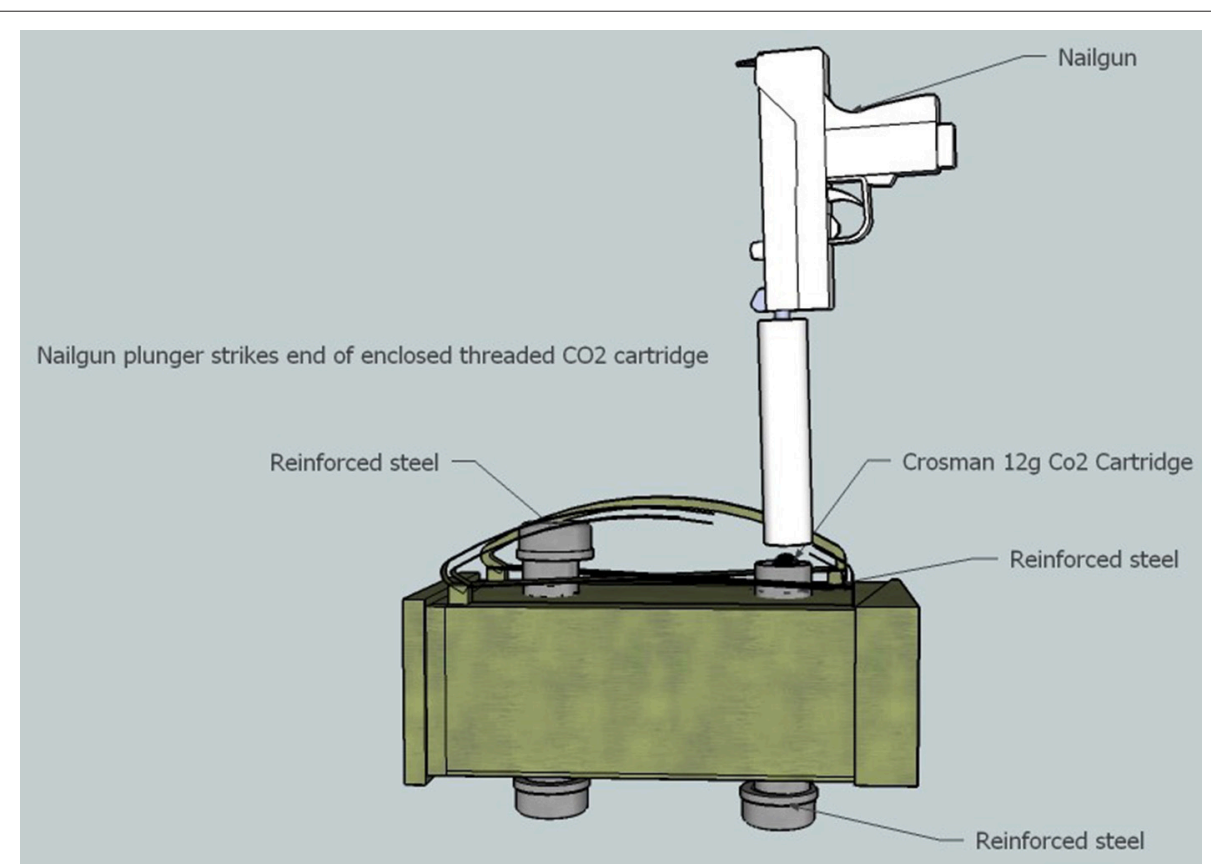

FIGURE 2 | Lateral view of blast chamber. When positioned within the chamber, the rounded end of the $\mathrm{CO}_{2}$ cartridge sticks up approximately $1 / 2 \mathrm{~cm}$ out of the chamber to engage with the nail-gun safety sleeve. The re-enforced steel caps allow access to the blast chamber before and after each blast.

pulse. The cartridges containing 10 and $15 \mathrm{cc}$ of blood were impacted, while the cartridge with $5 \mathrm{cc}$ of blood (control) was not. Following actuation of the nail gun, the blast chamber was turned over and the steel cartridge was carefully removed and placed into a test tube holder. Patient identifier sheets were double checked, and the time of blast was recorded.

\section{Blood Analysis}

Entire contents of cartridges were removed from the steel containers and transferred to polypropylene tubes for incubation at $37^{\circ} \mathrm{C}$. The entire volume in the container was transferred to minimize exposure to steel. At defined post-blast times (2, 4, 24, 48 , and $72 \mathrm{~h}$ ) the blood was analyzed by means of a Siemens 


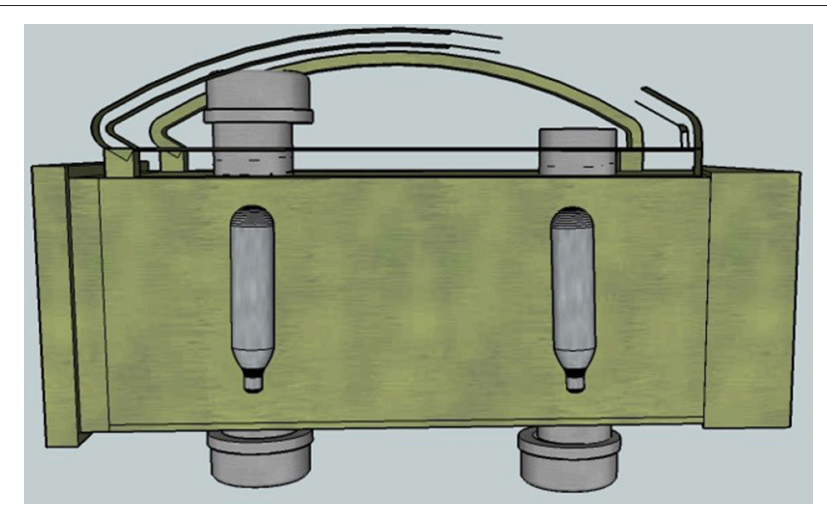

FIGURE 3 | Cut-away view showing the positioning of the steel $\mathrm{CO}_{2}$ cartridges inside the blast chambers. Two chambers were constructed to expedite the blasting process.

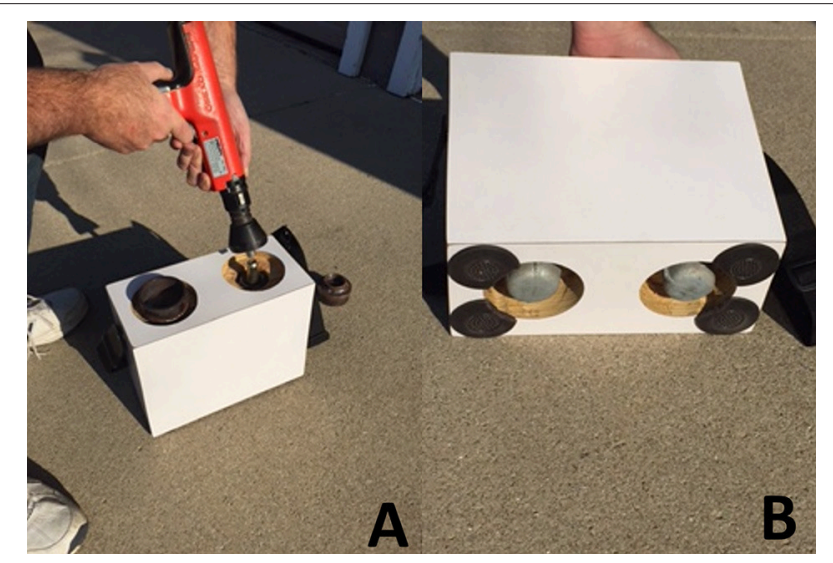

FIGURE 4 | (A) Image of final blast chamber and nail gun being positioned over a $\mathrm{CO}_{2}$ cartridge (B) Underside of final blast chamber underside showing access to the $\mathrm{CO}_{2}$ blasted cartridge.

Vista 1500. K-ABL was assayed in a blood gas analyzer using potentiometry, $\mathrm{LDH}$ with a substrate method, and plasma $\mathrm{HgB}$ using the sodium lauryl sulfate method (utilizing the Sysmex $\mathrm{XN}-3000)$ [12].

\section{Statistical Analysis}

Statistical analysis was conducted with SPSS statistical software (SPSS Inc., Chicago, IL). $\mathrm{HgB}$ and $\mathrm{LDH}$ distributions were skewed and thus analyzed before and after log transformation. It should be noted that $\mathrm{K}-\mathrm{ABL}$ had many readings of 10 , the upper limit of detection. Mixed ANOVAs with "time" $(2,4,24,48$, and $72 \mathrm{~h})$ as the within-subjects factor and "group" (slosh, no-slosh, and control) as the between-subjects factor were used to evaluate the effect of blood blasting on temporal changes in indicators of red cell damage $(\mathrm{HgB}, \mathrm{K}-\mathrm{ABL}$, $\mathrm{LDH})$, respectively, to determine whether there were statistically significant interactions using an alpha level of 0.05 selected $a$ priori.

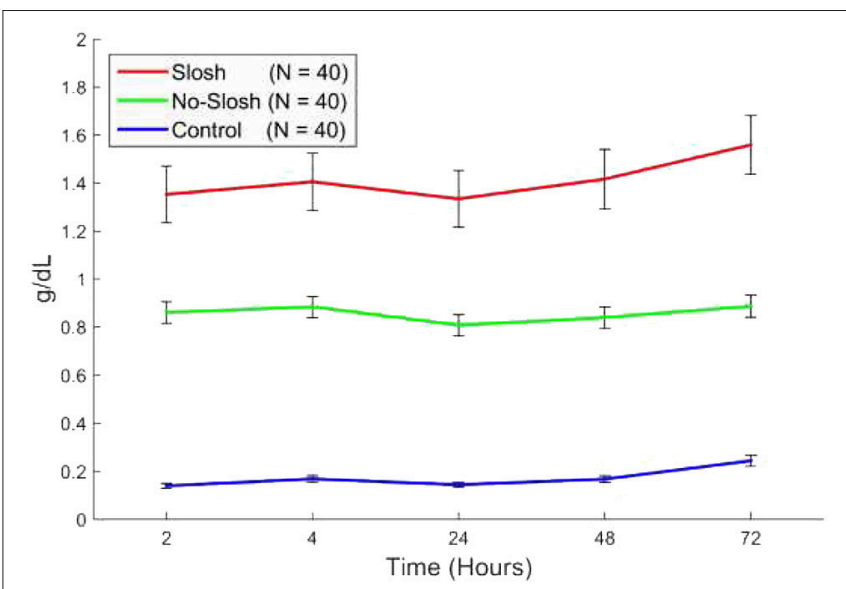

FIGURE 5 | Blood levels of HgB in "slosh," "no-slosh," and "control" groups, measured at 2, 4, 24, 48, and $72 \mathrm{~h}$ post-blast. '*' indicates statistically significant differences at the current time point relative to previous ones.

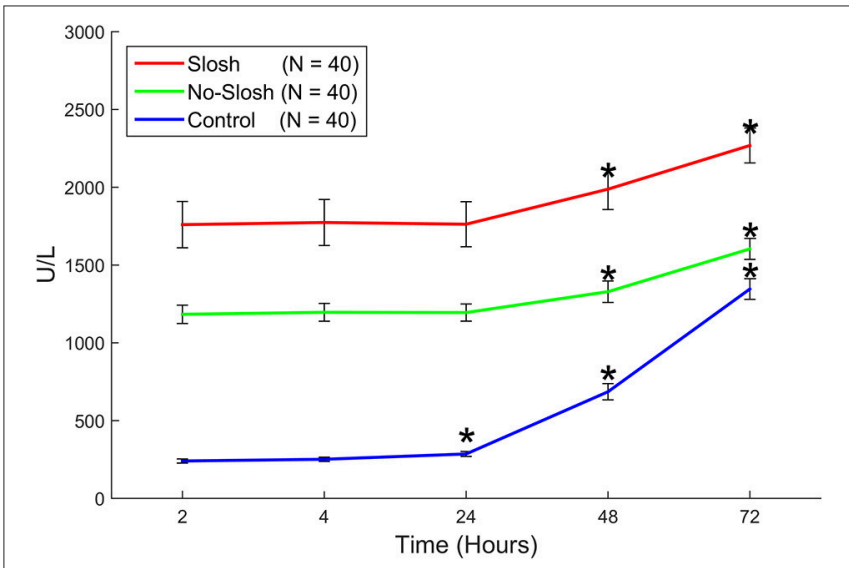

FIGURE 6 | Blood levels of LDH in "slosh," "no-slosh," and "control" groups, measured at 2, 4, 24, 48, and $72 \mathrm{~h}$ post-blast. ' "' indicates statistically significant differences at the current time point relative to previous ones.

\section{RESULTS}

\section{Hemoglobin}

There was a statistically significant group $\times$ time interaction on temporal changes in $\operatorname{HgB}(p<0.0001)$. There were no temporal differences in $\mathrm{HgB}$ concentration among groups over time ( $p=$ 0.40) (Figure 5).

\section{Lactate Dehydrogenase}

There was a statistically significant group $\times$ time interaction on temporal changes in $\mathrm{LDH}(p<0.0001)$. In the control group, there were significant main effects among all temporal change intervals (all $p<0.0001$ ) except for the interval $2-4 \mathrm{~h}$. In both the slosh and the no-slosh groups, there were significant main effects among all temporal change intervals (all $p<$ 0.0001 ) except for the intervals $2-4 \mathrm{~h}, 2-24 \mathrm{~h}$, and $4-24 \mathrm{~h}$ (Figure 6). 


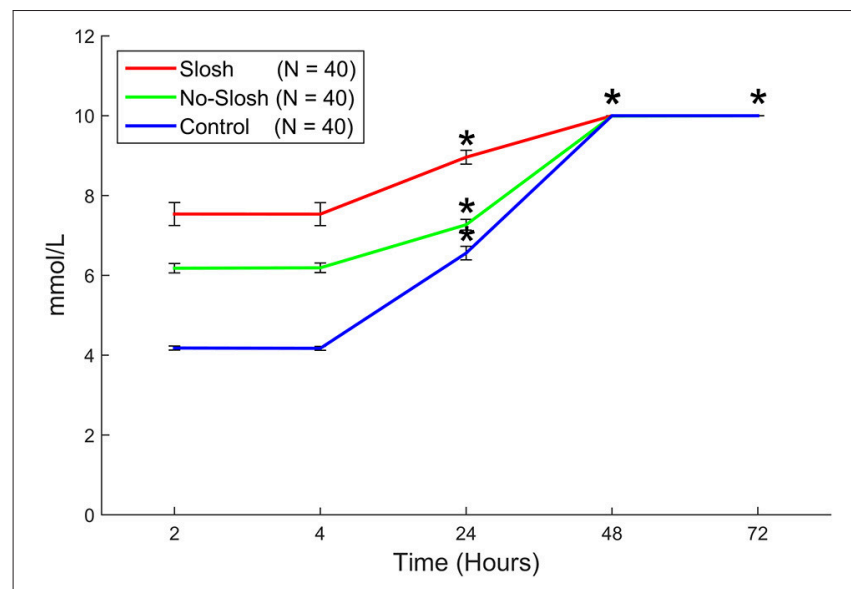

FIGURE 7 | Blood levels of $\mathrm{K}^{+}$(potassium) in "slosh," "no-slosh," and "control" groups, measured at 2, 4, 24, 48, and $72 \mathrm{~h}$ post-blast. " *' indicates statistically significant differences at the current time point relative to previous ones.

\section{Potassium}

There was a statistically significant group $\times$ time interaction $(p$ $<0.0001$ ) on temporal changes in K-ABL. In the control group, there were significant main effects among all temporal change intervals (all $p<0.0001$ ) except for the interval $2-4 \mathrm{~h}$. In both the slosh and the no-slosh groups, there were significant main effects among all temporal change intervals (all $p<0.0001$ ) except for the intervals $2-4 \mathrm{~h}$ and $48-72 \mathrm{~h}$ (Figure 7). In looking at each of the measures the most dramatic changes occurred in the first follow-up after blast (Figure 8).

\section{DISCUSSION}

Slosh theory is based on the physics of hydrodynamics and how moving fluids within containers can promote "inelastic" collisions over "elastic" ones (in-elastic collisions allow transfer of energies between colliding objects whereas elastic collisions reduces this energy transfer). From a red cell perspective, we interpret these data as a short-term response to blast energy followed by a delayed effect. We observe that free $\mathrm{HgB}$ rises the most in the first $2 \mathrm{~h}$. post-blast, but at that point, one would expect a flat trajectory if majority of the lysis occurred only on the initial blast. Blood will lyse over time on its own but the blasted samples seem to have accelerated this degree of free hemoglobin release over this time period. Figure 5 demonstrates the absolute rise in $\mathrm{HgB}$ (at $2 \mathrm{~h}$. post-blast), was $1.35-0.17=1.18$, while that at $72 \mathrm{~h}$ was $1.62-0.22=1.40$ (a $19 \%$ increase over $2 \mathrm{~h}$ data point). The immediate lysis is most likely due to membrane stress/strain due to the fluid displacement pulse and/or cavitation (the formation of vapor cavities with resultant implosion of the bubbles formed) within the vessels. The delayed effect could be osmotic, due to membrane ion transport changes with increased extracellular $\mathrm{K}^{+}$due to either hemolysis or leakage of $\mathrm{K}^{+}$from intact RBCs. Leakage of $\mathrm{K}^{+}$out of the cells may or may not be accompanied by leakage of $\mathrm{Na}^{+}$into the cells depending on the transport pathway involved. Yet another explanation of the delayed reaction could be due to the fact that mature red cells lack many the regenerative mechanics (mitochondria and Golgi apparatus) and processes to repair themselves, and thus, may not be able to sustain themselves, after a "lethal impact." Specifically, blast waves are known to produce large amounts of methemoglobin [13]. and this form of hemoglobin is unable to carry or release oxygen for the cell's neighboring tissues. As the inner red cell membrane is the major repository for methemoglobin reductase [14], which is necessary to make hemoglobin able to transport oxygen, and if the rate constant for conversion from methemoglobin back to hemoglobin is exceeded, then a furthering or cascading of events could be propagated.

The empty spaces in the cartridges (meant to model the freely moving venous blood in the cranium) foster transient velocity gradients, with resultant formation of vapor cavities, and upon implosion, ultimately result in cavitation. Flow velocity under the influence of a pressure pulse would depend on the ability of surrounding tissues to "give way" allowing voids in the fluids. Cavitation is dependent on formation of these voids which are harder to form in closed spaces due to the steep pressure vs volume relationship. This theory predicts the mechanism of energy impartation into the cerebrum (resulting in TBI) to be moving fluids, but it has not been determined which fluid in the cranium is most likely to be implicated, cerebral spinal fluid (CSF) or blood (and specifically, arterial or venous)? Arterial blood is more tightly "contained" and pressurized as the vessel walls themselves contain a muscle layer and are thus more stiff and non-compliant. Therefore, one would expect collisions with this arterial fluid space to be more elastic and result in a lesser net transfer of thermal, kinetic, vibrational or acoustic energy. In comparison, venous blood is under a lower pressure and cranial veins have no valves to prevent flow in either direction and the vessels themselves are not stiff but distensible by up to $25 \%$ [5], and are even referred to as venous capacitance vessels in many cases. Tissues or fluids of differing densities/shear will accelerate and decelerate at different rates, thus if impacted with a concussive wave, in the case of blood, which alters its shear at tissue boundaries, one would expect a facilitation of separation of these boundaries, i.e., vapor cavities (or cavitation).

Thus, impacting energies entering venous blood would follow a more in-elastic collision model whereby there is a greater transference of thermal, kinetic, vibrational or acoustic energy to the tissues, and subsequent deformation. As there was still evidence of hemolysis over the control group, containment of the moving fluids with these cartridges (and by inference the cranium) would serve to mitigate energy transfer and potential damage, but not prevent it entirely. Specifically, we contend that blood, itself, could be a link in the chain of the pathophysiology of TBI. If blood can be made to cavitate by externally imparted energies, and this cavitation can damage not only the blood but the surrounding vessels and tissues, then finding a way to mitigate this energy impartation into blood may reduce the degree of incidence of blast imparted brain injury. As there were no significant hemolysis differences between the sexes this data would seem to support that there is little gender difference in the mechanical properties of blood. 

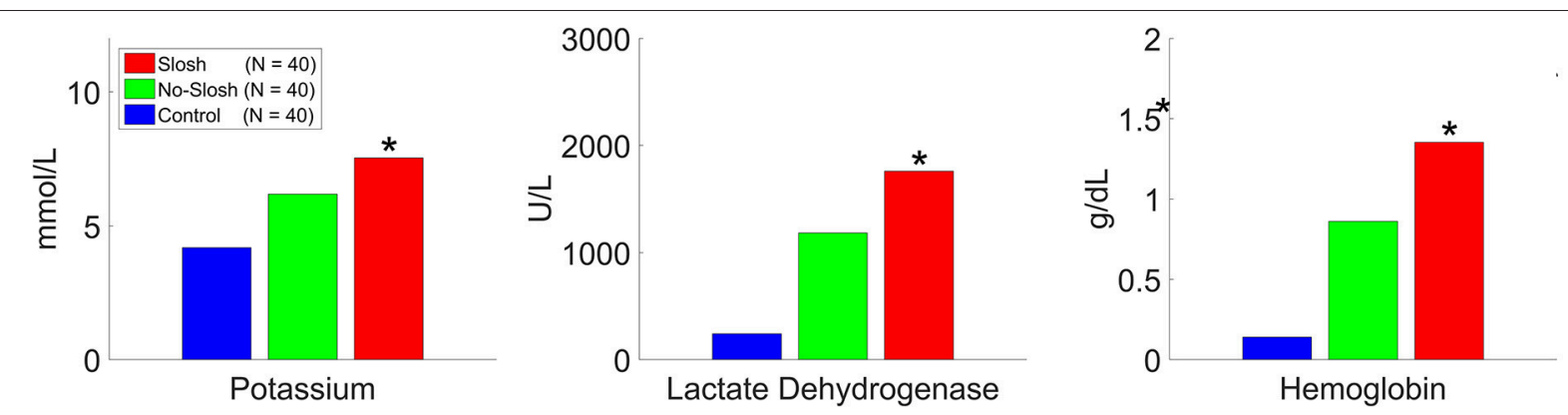

FIGURE 8 | Potassium, lactate dehydrogenase, and potassium levels as measured $2 \mathrm{~h}$ following the blood blasting procedure, with BLUE representing "control”, GREEN representing "no-slosh," and RED representing "slosh." "*” indicates statistically significant difference.

Perhaps just as importantly, when the cranium is impacted (by blunt trauma or blast wind), the bones of the skull are coupled in such a way that they will be "pushed" faster than the fluids within. Since there are no valves in the venous system, the trailing side of the cerebrum should squeeze blood between it and the skull and should expel venous blood out and down the low resistance outflow tracts. The leading side of the cranial acceleration would tend to pull venous blood into the venous capacitance vessels and sinuses (since the brain lags behind the skull). The rapid "pulling" of fluids creates a vacuum space which in turn would promote vapor cavities, and thus mimics the necessary parameters to promote "cavitation". Cavitation has been a known entity in discussing the pathophysiology of TBI since first described by Gross in 1958 [15] and subsequent investigators [16-20], but nearly all of these studies may have been limited by not providing a compliant vessel as the model of study (or by using water instead of actual blood).

Science has struggled with explaining how the physics of a blast wave can impart the chemistry that is seen within the cranium. Cavitation, as a theory, does fill this void. "Cavitation implosion produces the temperature of the sun, the pressure at the bottom of the ocean, the lifetime of a lightning strike, and a million times faster cooling than a red-hot iron rod plunged into water. It can increase chemical reactivity by nearly a million-fold" [21]. Further, upon cavitation bubble implosion, the collapse is usually asymmetrical leading to the formation of microjets of fluid directed toward the boundary (vessel walls) moving at speeds upward of $100 \mathrm{~m} / \mathrm{s}$. [22] The shock and pressure differentials generated by the impact of the microjet developed during bubble collapse close to the boundary may result in the damage to the integrity of that wall, possibly the blood brain barrier, or even the brain matter behind it.

Blood manifests certain characteristics distinct from most other fluids, including CSF. Blood behaves in a non-Newtonian, shear-thinning manner in the setting of applied external shear (or impact forces) [23]. This means that upon force impartation the fluids within a container such as blood within the crania are more prone to accelerate away from each other rather than forming bonds within or creating micro clusters as they do in shear thickening fluids (used in liquid body armor). Given that cavitation is the formation of vapor cavities that then implode, blood is predicted to more easily cavitate than water or CSF. This premise is supported by Bertram et al. (unpublished report), whereby Mat lab calculations predicted cavitation in cranial veins with as little as $85 \mathrm{x} g$ force rather than the collective investigators above estimating a needed force of nearly $400 \mathrm{x} \mathrm{g}$.

Cavitation inside the human blood stream is not a novel idea. In fact, mankind has been using shock wave lithotripsy for decades and most of the known complications of imparting high energy ultrasound waves (lithotripsy) is known to be as a result of cavitation and the resultant platelet aggregation, complementactivation, fibrinolysis, free radicals, [24] release of tissue-factor and endothelial damage [25]. Interestingly, these lithotripterinduced cavitation complications seem to suggest similarity to those footprints of TBI injury markers of the cranium [26].

Hence, this study of blood being subjected to IED levels of force impartation (150 $\mathrm{kPa}$ of pressure imparted over a brief period of only 3-4 $\mu$ s) [11] should serve to test whether biological tissues (blood in this instance) can be damaged by IED blasts and that damage can be followed by observation of the signs of blood lysis (with resultant extravasation of $\mathrm{K}^{+}, \mathrm{LDH}$, and $\mathrm{HgB}$ ).

"Blood is uniquely susceptible to injury (especially blast) in the body due to fact that red cells lose their nucleus, mitochondria, and ribosomes, and thus, mature erythrocytes are less able to carry out oxidative phosphorylation and protein synthesis for repair" [27]. However, they still must sustain an active metabolism to maintain the flexibility and integrity of the red cell membrane as well as to preserve hemoglobin in its functional form to ensure adequate oxygen delivery [28]. As mentioned, past investigators have postulated that higher order blast waves impart their damaging effects through blood cavitation, a purported mechanism of TBI dating back to 1958 [15]. This theory was ultimately upended after investigators were unable to reproduce cavitation in the lab without imparting large g-forces $(>400 \mathrm{x}$ g). But now, a new understanding has come to light about the promoters of cavitation in blood that may provide a rethinking of Shear/Strain Theory as the most accepted pathophysiology mechanism of TBI in deference to cavitation. At a minimum, the present study should help to redirect investigations of IED effects on human tissue to include the blood itself and possibly reintroduce cavitation as the more likely mechanistic (or at least co-mechanistic) view of TBI. 


\section{Limitations of This Study}

We were not able to foresee the dramatic release or leaking of potassium from all the cells in both groups. We did not dilute the samples at $48 \mathrm{~h}$ to better approximate the final potassium serum levels and they all registered as $>10$. Second, it would have been illustrative to have measured the time course of these released cellular constituents within the first 5-10 min post-blast exposure to examine to what degree the red blood cells immediately lyse upon blast or if there is a time delay before the onset of lysis. Lastly, as the vast majority of the free hemoglobin was released by the $2 \mathrm{~h}$ post-blast timepoint, we cannot be assured of the significance of the roughly 19\% increase thereafter being from an osmotic effect or true blast effect on the cells. Likewise the similar response in $\mathrm{LDH}$ and potassium demonstrated similar patterns and should be interpreted with caution relatively to the longitudinal time point measures.

\section{CONCLUSIONS}

IED blast level force imparted into cartridges of blood demonstrated red blood cell hemolysis over a 1 to 3-day time frame. Further, the containment of the blood (reducing slosh) by filling the test cartridges fully functioned to mitigate a significant amount of lysis. This mechanism of removing compliance and thus reducing the absorption of blast energy of the blood within a moving container has the potential of becoming a novel way of mitigating blood and surrounding brain tissue damage in the setting of tactical and military blast exposure. To the authors' knowledge, this represents the first attempt to implicate an intermediary of liquid blood rather than just solid brain tissue as a possible pathway for the pathophysiologic changes of TBI. "Cavitation implosion produces the temperature of the sun, the pressure at the bottom of the ocean, the lifetime of a lightning strike, and a million times faster cooling than a red-hot iron rod

\section{REFERENCES}

1. Beshore G. Science in Ancient. New York, NY: Franklin Watts (1998).

2. Tole NM, Ostensen H. Basic Physics of Ultrasonographic Imaging. Geneva: World Health Organization (2005).

3. Sawant R, Jathar S, Rajadhyaksha S, Kadam P. Red cell hemolysis during processing and storage. Asian J Trans Sci. (2007) 1:47. doi: 10.4103/0973-6247.33446

4. Turner RC, Naser ZJ, Bailes JE, Smith DW, Fisher JA, Rosen CL. Effect of slosh mitigation on histologic markers of traumatic brain injury: laboratory investigation. J Neurosurg. (2012) 117:1110-8. doi: 10.3171/2012.8.JNS12358

5. Leach JL, Smith DW, Myer GD. Mild neck compression alters intracranial venous sinus volume: implications for a novel neuroprotective effect in concussion. In: American Society of Neuroradiology Annual Meeting. San Diego, CA (2013).

6. Myer GD, Yuan W, Barber Foss KD, Smith D, Altaye M, Krueger D, et al. The effects of external jugular compression applied during head impact exposure on longitudinal changes in brain neuroanatomical and neurophysiological biomarkers: a preliminary investigation. Front Neurol. (2016) 7:74. doi: 10.3389/fneur.2016.00074

7. Myer GD, Yuan W, Barber Foss KD, Thomas S, Smith D, Altaye M. et al. Analysis of head impact exposure and brain microstructure response in a season-long application of a jugular vein compression collar: a prospective, plunged into water. It can increase chemical reactivity by nearly a million-fold" [21].

If blood is cavitating at relatively lower impact levels (with the resultant release of its own shock wave, chemical cascades, and microjets into surrounding tissues, then mitigating the blood's ability to cavitate could abate the ravages of repetitive head impact and TBI. Further research into cavitation as a lead pathophysiologic mechanism of TBI is warranted and attempts to lessen cavitation at the fluid level of the cranium should be implemented.

\section{ETHICS STATEMENT}

This study was carried out in accordance with the recommendations of The Institutional Review Board (IRB) at Cincinnati Children's Hospital Medical Center with written informed consent from all subjects. All subjects gave written informed consent in accordance with the Declaration of Helsinki. The protocol was approved by The Institutional Review Board (IRB) at Cincinnati Children's Hospital Medical Center.

\section{AUTHOR CONTRIBUTIONS}

DS, RF, and DKS contributed to study design, manuscript development, data interpretation, and final review. CD and GM contributed to study design, manuscript development, data analysis, and final review. CM and QS contributed to study design, manuscript development and final review.

\section{ACKNOWLEDGMENTS}

The authors acknowledge Reid Health (Richmond, Indiana): Providing laboratory and blood blasting site support. neuroimaging investigation in American football. Br J Sports Med. (2016) 50:1276-85. doi: 10.1136/bjsports-2016-096134

8. Smith DW, Bailes JE, Fisher JA, Robles J, Turner RC, Mills JD. Internal jugular vein compression mitigates traumatic axonal injury in a rat model by reducing the intracranial slosh effect. Neurosurgery (2012) 70:740-6. doi: 10.1227/NEU.0b013e318235b991

9. Koseoglu M, Hur A, Atay A, Cuhadar S. Effects of hemolysis interference on routine biochemistry parameters. Biochem Med. (2011) 21:79-85. doi: 10.11613/BM.2011.015

10. Masel BE, Bell RS, Brossart S, Grill RJ, Hayes RL, DeWitt DS. Galveston Brain Injury Conference 2010: clinical and experimental aspects of blast injury. J Neurotrauma (2012) 29:2143-71. doi: 10.1089/neu. 2011.2258

11. DeWitt DS, Perez-Polo R, Hulsebosch CE, Dash PK, Robertson CS Challenges in the development of rodent models of mild traumatic brain injury. J Neurotrauma (2013) 30:688-701. doi: 10.1089/neu. 2012.2349

12. Goyal T, Schmotzer CL. Validation of hemolysis index thresholds optimizes detection of clinically significant hemolysis. Am J Clin Pathol. (2015) 143:57983. doi: 10.1309/AJCPDUDE1HRA0YMR

13. Yazbeck-Karam VG, Aouad MT, Kaddoum RN, Baraka AS. Methemoglobinemia after a blast injury. J Am Soc Anesthesiol. (2004) 100:448-9. doi: 10.1097/00000542-200402000-00040 
14. Choury D, Leroux, A, Kaplan JC. Membrane-bound cytochrome b5 reductase (methemoglobin reductase) in human erythrocytes. Study in normal and methemoglobinemic subjects. J Clin Invest. (1981) 67:149. doi: 10.1172/JCI110007

15. Gross AG. A new theory on the dynamics of brain concussion and brain injury. J Neurosurg. (1958) 15:548-61. doi: 10.3171/jns.1958.15.5.0548

16. Kurosawa Y, Kato K, Saito S, Kubo M, Uzuka T, Fujii Y, et al. Basic study of brain injury mechanism caused by cavitation. Conf Proc IEEE Eng Med Biol Soc. (2009) 2009:7224-7. doi: 10.1109/IEMBS.2009.53 35260

17. Liu S, Yin Z, Zhao H, Yang G. Investigation of the cavitation and pressure change of brain tissue based on a transparent head model in its decelerating impact. J Mech Med Biol. (2010) 10:361-72. doi: 10.1142/S02195194100 03447

18. Wardlaw A, Goeller J. Cavitation as a possible traumatic brain injury (TBI) damage mechanism. In: 26th Southern Biomedical Engineering Conference SBEC 2010. College Park, MA:Springer (2010).

19. Nakagawa A, Manley GT, Gean AD, Ohtani K, Armonda R, Tominaga $\mathrm{T}$, et al. Mechanisms of primary blast-induced traumatic brain injury: insights from shock-wave research. J Neurotrauma (2011) 28:1101-19. doi: 10.1089/neu.2010.1442

20. Goeller J, Wardlaw A, Treichler D, O'Bruba J, Weiss G. Investigation of cavitation as a possible damage mechanism in blast-induced traumatic brain injury. J Neurotrauma (2012) 29:1970-81. doi: 10.1089/neu. 2011.2224

21. Suslick KS. The Chemistry of Ultrasound. Encyclopaedia Britannica. Chicago (1994). p 138-155.

22. Brujan E. The role of cavitation microjets in the therapeutic applications of ultrasound. Ultrasound Med Biol. (2004) 30:381-7. doi: 10.1016/j.ultrasmedbio.2003.10.019
23. Comiskey P, Yarin A, Kim S, Attinger D. Prediction of blood back spatter from a gunshot in bloodstain pattern analysis. Phys Rev Fluids (2016) 1:043201. doi: 10.1103/PhysRevFluids.1.043201

24. Edmonds PD, Sancier KM. Evidence for free radical production by ultrasonic cavitation in biological media. Ultrasound Med Biol. (1983) 9:635-9. doi: 10.1016/0301-5629(83)90009-1

25. Rodriguez RA, Ruel M, Labrosse M, Mesana, T. Transcranial doppler and acoustic pressure fluctuations for the assessment of cavitation and thromboembolism in patients with mechanical heart valves. Interact Cardiovasc Thorac Surg. (2008) 7:179-83. doi: 10.1510/icvts.2007.167569

26. Woodcock T, Morganti-Kossmann MC. The role of markers of inflammation in traumatic brain injury. Front Neurol. (2013) $4: 18$. doi: 10.3389/fneur.2013.00018

27. Zhang ZW, Cheng J, Xu F, Chen YE, Du JB, Yuan S. et al. Red blood cell extrudes nucleus and mitochondria against oxidative stress. Iubmb Life (2011) 63:560-5. doi: 10.1002/iub.490

28. Prchal JT, Gregg XT. Red Cell Enzymes. ASH Educ Prog Book (2005) 2005:1923. doi: 10.1182/asheducation-2005.1.19

Conflict of Interest Statement: The authors declare that the research was conducted in the absence of any commercial or financial relationships that could be construed as a potential conflict of interest.

Copyright (c) 2018 Smith, Franco, DiCesare, Schneider, McGill, Smith and Myer. This is an open-access article distributed under the terms of the Creative Commons Attribution License (CC BY). The use, distribution or reproduction in other forums is permitted, provided the original author(s) and the copyright owner(s) are credited and that the original publication in this journal is cited, in accordance with accepted academic practice. No use, distribution or reproduction is permitted which does not comply with these terms. 